\title{
FOCUSING WAVES IN UNKNOWN MEDIA BY MODIFIED TIME REVERSAL ITERATION
}

\author{
MATIAS DAHL, ANNA KIRPICHNIKOVA, AND MATTI LASSAS
}

\begin{abstract}
We study the wave equation in a bounded domain or on a compact Riemannian manifold with boundary. Assume that we are given the hyperbolic Neumann-to-Dirichlet map on the boundary corresponding to physical boundary measurements. We consider how to focus waves, that is, how to find Neumann boundary values so that at a given time the corresponding wave converges to a delta distribution $\delta_{y}$ while the time derivative of the wave converges to zero. Such boundary value are generated by an iterative sequence of measurements. In each iteration step we apply time reversal and other simple operators to measured data and compute boundary values for the next iteration step. The key feature of the algorithm is that it does not require knowledge of the coefficients in the wave equation, that is, the material parameters inside the media. However, we assume that the point $y$ where the wave focuses is known in travel time coordinates.
\end{abstract}

Keywords: Focusing of waves, wave equation, time reversal.

\section{INTRODUCTION}

Let us consider the wave equation in a bounded domain $M$,

$$
\left\{\begin{array}{l}
u_{t t}(x, t)+\mathcal{A} u(x, t)=0, \quad \text { in } M \times \mathbb{R}_{+}, \\
\left.u\right|_{t=0}=0,\left.\quad u_{t}\right|_{t=0}=0 \\
\left.\partial_{\nu} u\right|_{\partial M \times \mathbb{R}_{+}}=f
\end{array}\right.
$$

where $A$ is a 2 nd order elliptic partial differential operator.

In this paper we show how to construct Neumann boundary values $f$ such that at time $T$, the wave $\left(u(T), u_{t}(T)\right)$ is arbitrarily close to $\left(c \delta_{y}, 0\right)$, where $\delta_{y}$ is the Dirac delta distribution at a chosen point $y \in M$. We call such waves focusing waves. To find such boundary values, we only assume that we can make physical measurements from the boundary of $M$. For given Neumann boundary values we can measure the Dirichlet boundary values of the wave. A focusing wave can then be generated by an iterative sequence of measurements. In each iteration step we apply time reversal and other simple operators to measured data and compute boundary values for the next iteration step. 
The iteration algorithm in this paper is closely related to time reversal methods. Let us therefore shortly discuss the underlying idea and the usually used approximations behind these methods. As a simple example, let us consider a domain $M$ in $\mathbb{R}^{3}$, and suppose that we can measure waves and generate sources on the boundary of $M$. Let us first assume that there is a theoretical point source at $y \in M$, and we measure the wave and/or its normal derivative at the boundary of $M$. Assume further that we record this signal, reverse it in time, and re-emitted into $M$, see [19]. Then one can show (assuming certain approximations hold, see [19, 20, 16]), that the re-emitted wave will travel like the original wave, but as if time were running backwards. This causes the re-emitted wave to focus near $y$.

This principle can also be used for imaging. To find a small scatterer $D$ in a relatively homogeneous domain $M$, one sends a wave into $M$. If the scatterer is small and the single scattering approximation is justified, the scattered wave corresponds to a wave produced by a point source at $D$. If we record this scattered signal at the boundary, reversed it in time, and re-emit it into the domain, it will focus at the scatterer. Furthermore, this focusing has been observed to be quite stable under perturbations of the medium. Thus, if the re-emitted wave is simulated (by computational means) in homogeneous media, it will focus at the location of $D$. In this way a small scatterer can be found using relatively simple computational methods. The above basic idea has been refined in various ways. If the target area contains multiple scatterers, an iteration scheme can be used to focus the wave on any of the scatterers 33 .

Besides imaging, time reversal can be used to focus a wave onto a scatterer, say, inside the human body. One application of this is litotripsy, where one breaks down a kidney or bladder stone using a focusing ultrasonic wave. Another application is hyperthermia, where a cancer is destroyed by an excessive heat dose generated by a focusing wave. Let us point out that for the wave equation, there are various methods to estimate material parameters in travel time coordinates from boundary measurements. These methods are, however, quite unstable [2, 25]. Therefore they might not be suitable for hyperthermia, where safety is crucial. An important question is therefore how to focus waves in unknown media.

For reviews and extensions on time reversal, see seminal papers of $\mathrm{M}$. Fink, [17, 18, 20]. Time reversal methods have been intensively studied in random heterogeneous media where the statistic of the random media is known, see e.g. [5, 6, 7, 12, 13]. For time reversal in chaotic cavities, see [39]. For related analysis on time reversal methods, see also [3, 4, 15, 30, 34.

Let us describe the key features of the algorithm in this paper. First, to focus a wave we do not require knowledge of the material parameters 
inside the media. We only assume that the coordinates of the point are known in travel time coordinates. This means that focusing can be done in the same coordinates in which imaging is done. Thus, as the algorithm for focusing does not require media parameters obtained from imaging, errors in imaging do not accumulate into errors in focusing. Second, the algorithm can focus waves near an area having no scatterers. Third, the algorithm is computationally cheap. In a sense, all computations are done in the media; there is no need to solve the wave equation, of [23]. We will assume that the medium is linear, non-dispersive, non-dissipative, and frequency-independent. However, we do not need any other approximations like the single scattering approximations to prove that the algorithm works.

The limitations of the present algorithm is that we assume selfadjointness of operator $\mathcal{A}$ and that time $T$ is large enough. Moreover, the point $y$ where the wave focuses need to be specified in travel time coordinates unless operator $\mathcal{A}$ is known.

The present work is a continuation of [11] where a similar iterative scheme was introduced, for which $u(T)$ focuses to a delta-distribution, but the time derivative $u_{t}(T)$ is uncontrolled. The present work can also be seen as also a generalization of so-called retrofocusing in control theory, where the aim is to produce boundary sources giving the same final state as a boundary sources sent before in the medium, see [24, 31. The methodology in this paper arises from boundary control methods used to study inverse problems in hyperbolic equations [8, 9, 10, 26, 27, 28, 29].

The outline of this work is as follows. In Section 2 we introduce notation and review some relevant results from control theory. We also define the boundary operators that are needed in the iteration scheme. In Section 3 we describe the main results (Theorems 5 and 7 ) and outline their proofs, and in Section 4 we prove these results.

\section{Definitions}

We assume that $M \subset \mathbb{R}^{m}(m \geq 1)$ is the closure of an open $C^{\infty}$-smooth bounded set with non-empty smooth boundary $\partial M$ or a $C^{\infty}$-smooth compact manifold with boundary. Furthermore, we assume that $M$ is equipped with a $C^{\infty}$-smooth Riemannian metric $g=\sum_{j k} g_{j k} d x^{j} \otimes d x^{k}$. Elements of the inverse matrix of $g_{i j}$ are denoted by $g^{i j}$. Let $\mathrm{d} V_{g}$ be the smooth measure

$$
\mathrm{d} V_{g}=|g(x)|^{1 / 2} d x^{1} \wedge \cdots \wedge d x^{m},
$$

where $|g|=\operatorname{det}\left(\left[g_{j k}\right]\right)$. Then $L^{2}(M)$ is defined by the inner product

$$
\langle u, v\rangle=\int_{M} u(x) v(x) \mathrm{d} V,
$$


where $\mathrm{d} V=\mu \mathrm{d} V_{g}$ and $\mu \in C^{\infty}(M)$ is a fixed strictly positive function on $M$.

In wave equation (1), we assume $\mathcal{A}$ represents the most general formally selfadjoint elliptic partial differential operator with respect to the above inner product [27]. In local coordinates, $\mathcal{A}$ has the form

$$
\mathcal{A} v=-\sum_{j, k=1}^{m} \frac{1}{\mu(x)|g(x)|^{1 / 2}} \frac{\partial}{\partial x^{j}}\left(\mu(x)|g(x)|^{1 / 2} g^{j k}(x) \frac{\partial v}{\partial x^{j}}\right)+q(x) v,
$$

where $q$ is a smooth function $q: M \rightarrow \mathbb{R}$. For example, if $\mu=1$ and $q=0$ then $\mathcal{A}$ reduces to the Riemannian Laplace operator. Let us point out that $\mathcal{A}$ represents media that is linear, non-dissipative, non-dispersive, and frequency-independent.

On the boundary, operator $\partial_{\nu}$ is defined by

$$
\partial_{\nu} v=\sum_{j=1}^{m} \mu(x) \nu^{j} \frac{\partial}{\partial x^{j}} v(x)
$$

where $\nu(x)=\left(\nu^{1}, \nu^{2}, \ldots, \nu^{m}\right)$ is the unit interior normal vector satisfying $\sum_{j, k=1}^{m} g_{j k} \nu^{j} \nu^{k}=1$. To integrate functions on $\partial M$ we use the measure $\mathrm{d} S$ on $\partial M$ induced by $\mathrm{d} V_{g}$. If $B \subset \partial M \times \mathbb{R}_{+}$, we define

$$
L^{2}(B)=\left\{f \in L^{2}\left(\partial M \times \mathbb{R}_{+}\right): \operatorname{supp}(f) \subset B\right\}
$$

identifying functions and their zero continuations.

With these assumptions, the wave equation has a solution whenever $f \in L^{2}\left(\partial M \times \mathbb{R}_{+}\right)$, and we denote this solution by $u^{f}$. The map $f \mapsto u^{f}$ is linear over $\mathbb{R}$, and $\partial_{t} u^{f}=u^{\partial_{t} f}$ when $f, \partial_{t} f \in L^{2}\left(\partial M, \mathbb{R}_{+}\right)$.

Let $d(x, y)$ be the geodesic distance corresponding to $g$. The metric $d$ is also called the travel time metric because it describes how solutions to equation (1) propagate. By the finite velocity of wave propagation, (see [22]) we have that if $\Gamma \subset \partial M$ is open, and $f \in L^{2}\left(\Gamma \times \mathbb{R}_{+}\right)$, then at time $t>0$, solution $u^{f}$ is supported in the domain of influence

$$
M(\Gamma, t)=\{x \in M: \mathrm{d}(x, \Gamma) \leq t\} .
$$

The diameter of $M$ is defined as

$$
\operatorname{diam}(M)=\max \{\mathrm{d}(x, y): x, y \in M\} .
$$

The characteristic function of a set $S$ is denoted by $\chi_{S}$.

2.1. Controllability for wave equation. The seminal result implying controllability is Tataru's unique continuation result [35, 37].

Proposition 1 (Tataru). Let $u \in H_{\mathrm{loc}}^{1}\left(M \times \mathbb{R}_{+}\right)$be a solution of the wave equation

$$
u_{t t}(x, t)+\mathcal{A} u(x, t)=0 .
$$

Assume that

$$
\left.u\right|_{\Gamma \times(0,2 \tau)}=0,\left.\quad \partial_{\nu} u\right|_{\Gamma \times(0,2 \tau)}=0,
$$


where $\Gamma \subset \partial M$ is an non-empty open set and $\tau>0$. Then

$$
u(x, \tau)=0, \partial_{t} u(x, \tau)=0 \text { for } x \in M(\Gamma, \tau) .
$$

Using Tataru's unique continuation result, one can prove the following controllability results (The proof is postponed to section 4):

Proposition 2 (Approximate global controllability). If $T>\operatorname{diam}(M)$, then the linear subspace

$$
\left\{\left(u^{f}(T), u_{t}^{f}(T)\right): f \in C_{0}^{\infty}\left(\partial M \times \mathbb{R}_{+}\right)\right\}
$$

is dense in $H^{1}(M) \times L^{2}(M)$.

This result yields the following controllability result, see e.g. 27] and references therein.

Proposition 3 (Approximative local controllability). Let $\tau>0$, let $\Gamma_{1}, \ldots, \Gamma_{J} \subset \partial M$ be open non-empty sets, and let $0<s_{k}<\tau, k=$ $1, \ldots, J$. Suppose

$$
B=\bigcup_{j=1}^{J} \Gamma_{j} \times\left(\tau-s_{j}, \tau\right),
$$

and $P$ is multiplication by the characteristic function $\chi_{B}$,

$$
\begin{aligned}
P: L^{2}(\partial M \times(0, \tau)) & \rightarrow L^{2}(\partial M \times(0, \tau)), \\
f(x, t) & \mapsto \chi_{B}(x, t) f(x, t) .
\end{aligned}
$$

Then the linear subspace

$$
\left\{u^{P h}(\tau): h \in L^{2}(\partial M,(0, \tau))\right\}
$$

is dense in $L^{2}(N)$, where $N=\bigcup_{j=1}^{J} M\left(\Gamma_{j}, s_{j}\right)$.

2.2. Operators for boundary sources. In this section we introduce operators for manipulating boundary sources. These will be needed both in the proof of the main result and in the iteration scheme.

For initial boundary value problem (1) we define the non-stationary Neumann-to-Dirichlet map (or response operator) $\Lambda$ by setting

$$
\Lambda f=\left.u^{f}\right|_{\partial M \times \mathbb{R}_{+}}, \quad f \in L^{2}\left(\partial M \times \mathbb{R}_{+}\right) .
$$

In other words, we solve the wave equation (11) for a boundary source $f$, and measure boundary values for the solution $u^{f}$ when $t>0$. In this work, we only need the finite time Neumann-to-Dirichlet map,

$$
\Lambda_{2 T} f=\left.u^{f}\right|_{\partial M \times(0,2 T)},
$$

where $T>0$. By [36] the map

$$
\Lambda_{2 T}: L^{2}(\partial M \times(0,2 T)) \rightarrow H^{1 / 3}(\partial M \times(0,2 T))
$$

is bounded, where $H^{s}(\partial M \times(0,2 T))$ is the Sobolev space on $\partial M \times$ $(0,2 T)$. 
For $f \in L^{2}(\partial M,(0,2 T))$, let

$$
\begin{aligned}
R_{2 T} f(x, t) & =f(x, 2 T-t), \\
J_{2 T} f(x, t) & =\int_{[0,2 T]} J_{2 T}(s, t) f(x, s) d s,
\end{aligned}
$$

where $J_{2 T}(s, t)=\frac{1}{2} \chi_{L}(s, t)$ and

$$
L=\left\{(s, t) \in \mathbb{R}_{+} \times \mathbb{R}_{+}: t+s \leq 2 T, s>t\right\} .
$$

We call $R_{2 T}$ the time reversal map, and $J_{2 T}$ the time filter map [11]. On $L^{2}(\partial M \times[0,2 T])$ with the measure $d S(x) d t$, the adjoint of $\Lambda_{2 T}$ is [11,

$$
\Lambda_{2 T}^{*}=R_{2 T} \Lambda_{2 T} R_{2 T} .
$$

For $f \in L^{2}(\partial M,[0,2 T])$, let

$$
Q_{2 T} f=\int_{0}^{2 T} g(t, s) f(x, s) d s,
$$

be the time filter operator, where $g:(0,2 T)^{2} \rightarrow \mathbb{R}$,

$$
g(t, s)=\frac{1}{2\left(e^{4 T}-1\right)} \begin{cases}\left(e^{-t}+e^{t}\right)\left(e^{4 T} e^{-s}+e^{s}\right), & t<s, \\ \left(e^{-s}+e^{s}\right)\left(e^{4 T} e^{-t}+e^{t}\right), & t>s,\end{cases}
$$

is the Green's function for the problem

$$
\left\{\begin{array}{l}
\left(1-\partial_{t}^{2}\right) g(t, s)=\delta(t-s), \\
\left.\partial_{t} g\right|_{t=0}=0,\left.\quad \partial_{t} g\right|_{t=2 T}=0, \quad s \in(0,2 T) .
\end{array}\right.
$$

Next we consider $\Lambda_{2 T}, R_{2 T}, J_{2 T}, Q_{2 T}$ as operators such that

$$
\Lambda_{2 T}, \quad R_{2 T}, \quad J_{2 T}, \quad Q_{2 T}: L^{2}(\partial M \times[0,2 T]) \rightarrow L^{2}(\partial M \times[0,2 T]) .
$$

Below, we often denote $R_{2 T}, J_{2 T}$, and $Q_{2 T}$ by $R, J$, and $Q$. For $f, h \in L^{2}(\partial M \times[0,2 T])$ the Blagovestchenskii identity states that

$$
\int_{M} u^{f}(T) u^{h}(T) d V=\int_{\partial M \times[0,2 T]}(K f)(x, t) h(x, t) d S(x) d t,
$$

where $K: L^{2} \rightarrow L^{2}$ is the bounded operator

$$
K=K_{2 T}=R_{2 T} \Lambda_{2 T} R_{2 T} J_{2 T}-J_{2 T} \Lambda_{2 T} .
$$

For a proof, see e.g. [11]. The importance of this identity is that it shows that the inner product of solutions $u^{f}(T)$ and $u^{h}(T)$ can be calculated from the boundary. Namely, on the right hand side of the Blagovestchenskii identity, $d S$ is the Riemannian surface volume on $\partial M$, and $K$ is defined in terms of the Neumann-to-Dirichlet map $\Lambda_{2 T}$ and simple operators on boundary values like time reversal. The intrinsic Riemannian surface volume $d S$ on $\partial M$ is determined by $\Lambda_{2 T}$. Namely, by Tataru's unique continuation principle, the Schwartz kernel of $\Lambda_{2 T}$ is supported in

$$
E=\left\{\left(x, t, x^{\prime}, t^{\prime}\right) \in(\partial M \times[0,2 T])^{2}: t-t^{\prime} \geq d\left(x, x^{\prime}\right)\right\},
$$


and the boundary $\partial E$ is in the support. The set $\partial E$ determines the distances of points $z, z^{\prime} \in \partial M$ with respect to the intrinsic metric of the boundary $\left(\partial M, g_{\partial M}\right)$.

\section{ITERATIONS AND MAIN RESULTS}

3.1. Cutoff of wave. In this section we describe Theorem 5 which can be seen as a lemma used in the proof of Theorem 7 .

Let $X$ be the Banach space

$$
X=L^{2}(\partial M \times[0,2 T]) \times Y, \quad Y=H^{1}\left((0,2 T) ; L^{2}(\partial M)\right),
$$

such that the inner product on $X$ is

$$
\left\langle\left(\begin{array}{l}
h_{1} \\
a_{1}
\end{array}\right),\left(\begin{array}{l}
h_{2} \\
a_{2}
\end{array}\right)\right\rangle_{X}=\left\langle h_{1}, h_{2}\right\rangle_{L^{2}}+\left\langle a_{1}, a_{2}\right\rangle_{L^{2}}+\left\langle\partial_{t} a_{1}, \partial_{t} a_{2}\right\rangle_{L^{2}} .
$$

Definition 4. Let $T>2 \operatorname{diam}(M)$, and let

$$
B=\bigcup_{j=1}^{J} \Gamma_{j} \times\left(T-s_{j}, T\right),
$$

where $\Gamma_{1}, \ldots, \Gamma_{J} \subset \partial M$ are open non-empty sets, and $0<s_{k}<T$, $k=1, \ldots, J$. Let $P=\chi_{B}$ be the multiplication with the characteristic function of $B$ defined as in equation (2), and let $L: X \rightarrow X$ be the operator

$$
L=\left(\begin{array}{ll}
1 & 0 \\
0 & Q
\end{array}\right)\left(\begin{array}{cc}
2 P K P & -P K \\
-K P & K-\partial_{t} K \partial_{t}
\end{array}\right) .
$$

Let $\alpha \in(0,1)$, and let $\omega>0$ be such that $2\left(1+\|L\|_{X}\right)<\omega$, and let

$$
S=\left(1-\frac{\alpha}{\omega}\right) I-\frac{1}{\omega} L .
$$

If $f \in L^{2}\left(\partial M \times \mathbb{R}_{+}\right)$be a boundary source, we define a sequence $\left(\begin{array}{l}h_{n} \\ a_{n}\end{array}\right)=\left(\begin{array}{c}h_{j}(\alpha) \\ a_{j}(\alpha)\end{array}\right) \in X, n=1,2, \ldots$ by

$$
\left\{\begin{array}{l}
\left(\begin{array}{l}
h_{0} \\
a_{0}
\end{array}\right)=\frac{1}{\omega}\left(\begin{array}{c}
P K f \\
0
\end{array}\right), \\
\left(\begin{array}{l}
h_{n} \\
a_{n}
\end{array}\right)=\left(\begin{array}{l}
h_{0} \\
a_{0}
\end{array}\right)+S\left(\begin{array}{l}
h_{n-1} \\
a_{n-1}
\end{array}\right), \quad n=1,2, \ldots
\end{array}\right.
$$

Theorem 5 (Cutoff of wave). Let $a_{1}(\alpha), a_{2}(\alpha), \ldots$, be as in Definition 4. Then the sequence converges in $Y$,

$$
\lim _{n \rightarrow \infty} a_{n}(\alpha)=a(\alpha)
$$

and function $a(\alpha) \in Y$ on the right hand side satisfies

$$
\lim _{\alpha \rightarrow 0}\left(\begin{array}{c}
u^{a(\alpha)}(T) \\
u_{t}^{a(\alpha)}(T)
\end{array}\right)=\left(\begin{array}{c}
\chi_{N} u^{f}(T) \\
0
\end{array}\right),
$$


where both limits are in $L^{2}(M)$ and $N$ is the domain of influence

$$
N=\bigcup_{k=1}^{J} M\left(\Gamma_{k}, s_{k}\right) .
$$

Note that here $\omega$ may depend on $\alpha$. For instance, we can choose $\omega=1 / \alpha$.

Let us emphasize that the novelty of this theorem is the explicit iteration scheme for $a(\alpha)$ depending only on boundary measurements. The scheme depends on operators $J, Q, P$, and $K$ that can be calculated from the boundary of $M$. The first three are simple operators like integration and restriction. Operator $K=R_{2 T} \Lambda_{2 T} R_{2 T} J_{2 T}-J_{2 T} \Lambda_{2 T}$ involve time reversal $R_{2 T}$, time filtering $J_{2 T}$ and two evaluations of the Neumann-to-Dirichlet map $\Lambda_{2 T}$ which corresponds to two physical measurements. Hence, the first order approximation of $a(\alpha)$ requires 2 physical measurements. After that, each additional term requires 10 additional measurements. Thus, for finite approximation of $a(\alpha)$ we only need finitely many evaluations of the Neumann-to-Dirichlet map.

The full proof of Theorem 5 is given in Section 4.1. Let us here outline the main ideas. For $\alpha \in(0,1)$, boundary sources $h(\alpha), a(\alpha)$ are defined as the minimum of the functional

$$
\begin{aligned}
\mathcal{F}(h, a, \alpha)= & \left\|u^{f}(T)-u^{P h}(T)\right\|_{L^{2}(M)}^{2} \\
& +\left\|u^{P h}(T)-u^{a}(T)\right\|_{L^{2}(M)}^{2}+\left\|u_{t}^{a}(T)\right\|_{L^{2}(M)}^{2} \\
& +\alpha\left(\|h\|_{L^{2}(\partial M \times[0, T])}^{2}+\|a\|_{L^{2}(\partial M \times[0, T])}^{2}+\left\|\partial_{t} a\right\|_{L^{2}(\partial M \times[0, T])}^{2}\right),
\end{aligned}
$$

In the sequel, when there is no danger of misunderstanding, we denote the $L^{2}$-norms in the spaces $L^{2}(M), L^{2}(\partial M \times[0, T])$ etc. just by $\|\cdot\|$. In Lemma 8, we use convexity to prove that for each $\alpha$, there is a unique minimum $h(\alpha), a(\alpha)$, and by studying the Fréchet derivative of $\mathcal{F}$ we find a linear equation (equation (8) ) for this minimum. In Lemma 9 we show that iteration scheme (4) converges to a von Neumann sum that represents the solution to equation (8). That minimizer $a(\alpha)$ satisfies the sought limit is proven in Lemma 10. The key step in the proof is to use the approximative controllability results from Section 2.1 to show that the first terms in $\mathcal{F}$ can be arbitrarily close to $\|(1-$ $\left.\chi_{N}\right) u^{f}(T) \|^{2}$ and the next two terms can be made arbitrarily small.

3.2. Focusing of wave. To understand how one can focus waves using Theorem 5 suppose we have sets $B \subset \widetilde{B} \subset \partial M \times[0, T]$ (defined in terms of $\Gamma_{i}$ and $s_{i}$ as in Definition (4). Then Theorem 5 implies that 
there are boundary sources $a(\alpha)$ and $\widetilde{a}(\alpha)$ such that

$$
\begin{aligned}
& \lim _{\alpha \rightarrow 0}\left(\begin{array}{c}
u^{a(\alpha)}(T) \\
u_{t}^{a(\alpha)}(T)
\end{array}\right)=\left(\begin{array}{c}
\chi_{N} u^{f}(T) \\
0
\end{array}\right), \\
& \lim _{\alpha \rightarrow 0}\left(\begin{array}{c}
u^{\widetilde{a}(\alpha)}(T) \\
u_{t}^{\widetilde{a}(\alpha)}(T)
\end{array}\right)=\left(\begin{array}{c}
\chi_{\widetilde{N}} u^{f}(T) \\
0
\end{array}\right),
\end{aligned}
$$

where the domains of influences satisfy $N \subset \widetilde{N}$ at time $T$. As the solution operator $f \mapsto u^{f}$ is linear and commutes with $\partial_{t}$, solution $b(\alpha)=\widetilde{a}(\alpha)-a(\alpha)$ satisfies

$$
\lim _{\alpha \rightarrow 0}\left(\begin{array}{c}
u^{b(\alpha)}(T) \\
u_{t}^{b(\alpha)}(T)
\end{array}\right)=\left(\begin{array}{c}
\chi_{\widetilde{N} \backslash N} u^{f}(T) \\
0
\end{array}\right) .
$$

That is, in the limit, the solution corresponding to $b$ is supported in $\widetilde{N} \backslash N$. In the proof we construct $P$ and $\widetilde{P}$ such that $\widetilde{N} \backslash N$ is a family of sets that shrink onto a chosen point $\widehat{x}$. By further scaling $b$ with a suitable constant depending on the volume of $\widetilde{N} \backslash N$, we obtain the delta distribution.

To formulate the result, let us introduce some notation. By $\gamma_{x, \xi}$ we mean a geodesic in $(M, g)$ parametrized by the arclength such that $\gamma_{x, \xi}(0)=x, \dot{\gamma}_{x, \xi}(0)=\xi$, and $\|\xi\|_{g}=1$. Let $\nu=\nu(z), z \in \partial M$ be the interior unit normal vector to $\partial M$. Then there is a critical value $\tau(z)>0$, such that for $t<\tau(z)$ the geodesic $\gamma_{z, \nu}([0, t])$ is the unique shortest geodesic from its endpoint $\gamma_{z, \nu}(t)$ to $\partial M$, and for $t>\tau(z)$ it is no longer a shortest geodesic. We will not consider the degenerate case $t=\tau(z)$.

Definition 6. Let $T>2 \operatorname{diam}(M)$, let $\widehat{x}=\gamma_{\widehat{z}, \nu}(\widehat{T})$, where $\widehat{z} \in \partial M$, and $0<\widehat{T}<T$. Let $\Gamma_{j} \subset \partial M$ for $j=1,2, \ldots$ be open sets around $z$, such that $\Gamma_{j} \supset \bar{\Gamma}_{j+1}$ and $\bigcap_{j=1}^{\infty} \Gamma_{j}=\{\widehat{z}\}$.

Suppose $f \in C_{0}^{\infty}\left(\partial M \times \mathbb{R}_{+}\right)$. Let $a_{n}(\alpha, \varepsilon) \in Y$ be functions obtained from the iteration in Definition 4 when $B$ is the set

$$
B(\varepsilon)=\partial M \times(T-(\widehat{T}-\varepsilon), T),
$$

$\alpha \in(0,1)$, and $\varepsilon>0$ is sufficiently small. Similarly, let $a_{n}(\alpha, j, \varepsilon) \in$ $Y$ be functions obtained from the iteration in Definition 4 when $B$ is the set

$$
B(j, \varepsilon)=(\partial M \times(T-(\widehat{T}-\varepsilon), T)) \cup\left(\Gamma_{j} \times(T-(\widehat{T}+\varepsilon), T)\right),
$$

$\alpha \in(0,1), j=1,2, \ldots$ and $\varepsilon>0$ is sufficiently small.

Under these assumptions, let

$$
b_{n}(\alpha, j, \varepsilon)=\varepsilon^{-\frac{m+1}{2}}\left(a_{n}(\alpha, j, \varepsilon)-a_{n}(\alpha, \varepsilon)\right) \in Y .
$$

Theorem 7 is the main result of this paper. 
Theorem 7 (Focusing wave). Let $\widehat{z} \in \partial M, \widehat{T}, b_{n}(\alpha, j, \varepsilon)$ for $n=$ $1,2, \ldots$ be as in Definition $\left[\right.$. Then functions $b_{j}$ converge in $Y$,

$$
\lim _{n \rightarrow \infty} b_{n}(\alpha, j, \varepsilon)=b(\alpha, j, \varepsilon) \text {. }
$$

Moreover, if $\widehat{T}<\tau(\widehat{z})$ then functions $b(\alpha, j, \varepsilon) \in Y$ satisfy

$$
\lim _{\varepsilon \rightarrow 0^{+}} \lim _{j \rightarrow \infty} \lim _{\alpha \rightarrow 0^{+}}\left(\begin{array}{c}
u^{b(\alpha, j, \varepsilon)}(T) \\
u_{t}^{b(\alpha, j, \varepsilon)}(T)
\end{array}\right)=C(\widehat{x}) u^{f}(T, \widehat{x})\left(\begin{array}{c}
\delta_{\widehat{x}} \\
0
\end{array}\right)
$$

where the inner two limits are in $L^{2}(M)$ and the outer limit is in $\mathscr{D}^{\prime}(M)$, and $C(\widehat{x})$ is defined in equation (13).

If $\widehat{T}>\tau(\widehat{z})$, the limit (마) is zero.

Let us make three comments about this theorem. First, the condition $\widehat{T}<\tau(\widehat{z})$ means that $\widehat{z} \in \partial M$ is the closest boundary point to $\widehat{x}$ and $d(\widehat{x}, z)=d(\widehat{x}, \partial M)$. If a point $\hat{x}$ has a unique closest boundary point, we say that it is admissible. For example, on the closed disc, all points are admissible except the center. For a general $(M, g)$ the set of points that are not admissible has measure zero. Second, we assume that $f \in C_{0}^{\infty}\left(\partial M \times \mathbb{R}_{+}\right)$. Hence $u^{f} \in C^{\infty}\left(M \times \overline{\mathbb{R}}_{+}\right)$(see [32]), and $u^{f}(\widehat{x}, T)$ exists pointwise. Third, a function $v \in L^{2}(M)$ is interpreted as a distribution $v \in \mathscr{D}^{\prime}(M)$ by the formula

$$
\langle v, \phi\rangle=\int_{M} v \phi \mathrm{d} V, \quad \phi \in \mathscr{D}(M) .
$$

The delta distribution at $y \in M$ is defined by $\left\langle\delta_{y}, \phi\right\rangle=\phi(y)$ for $\phi \in \mathscr{D}(M)$.

\section{PROOFS}

We start with the proof of Proposition 2. The proof is a relatively direct consequence of Tataru's unique continuation theorem and can be found e.g. in the case of Dirichlet boundary conditions in [31, Lemma 2.1 .

Proof of Proposition 2. Assume that a pair

$$
(\psi,-\phi) \in\left(H^{1}(M) \times L^{2}(M)\right)^{\prime}=H_{0}^{-1}(M) \times L^{2}(M)
$$

satisfy the duality

$$
\left\langle u^{f}(T), \psi\right\rangle_{\left(H^{1}(M), H_{0}^{-1}(M)\right)}+\left\langle u_{t}^{f}(T),-\phi\right\rangle_{L^{2}(M)}=0
$$

for all $f \in C_{0}^{\infty}(\partial M \times(0, T))$. Note that $H^{1}(M)$ is the domain of the square root of the operator $\mathcal{A}+c I$ when $c$ is large enough, denoted by $\mathcal{D}\left(\mathcal{A}^{1 / 2}\right)$ and $H_{0}^{-1}(M)$ is the dual $H^{1}(M)=\mathcal{D}\left(\mathcal{A}^{-1 / 2}\right)$. Let

$$
\begin{aligned}
& e_{t t}+\mathcal{A} e=0 \quad \text { in } M \times(0, T), \\
& \left.\partial_{\nu} e\right|_{\partial M \times(0, T)}=0,\left.\quad e\right|_{t=T}=\phi,\left.\quad e_{t}\right|_{t=T}=\psi .
\end{aligned}
$$


By [32], $e \in C^{1}\left([0, T], L^{2}(M)\right) \cap C\left([0, T], H_{0}^{-1}(M)\right)$ and $\left.e\right|_{\partial M \times(0, T)} \in$ $H^{-2 / 5-\epsilon}(\partial M \times(0, T), \epsilon>0$. Thus we have in sense of distributions

$$
\begin{aligned}
0 & =\int_{M \times(0, T)}\left[u^{f}\left(e_{t t}+\mathcal{A} e\right)-\left(u_{t t}^{f}+\mathcal{A} u^{f}\right) e\right] d V d t \\
& =\int_{M}\left(u_{t}^{f}(T) \phi-u^{f}(T) \psi\right) d V+\int_{M \times(0, T)} f e d S_{x} d t \\
& =\int_{M \times(0, T)} f e d S_{x} d t
\end{aligned}
$$

for all $f \in C_{0}^{\infty}(\partial M \times(0, T))$. This yields that

$$
\left.e\right|_{\partial M \times(0, T)}=\left.\partial_{\nu} e\right|_{\partial M \times(0, T)}=0 .
$$

To apply unique continuation for $e \in C\left([0, T], H_{0}^{-1}(M)\right)$, let $\epsilon>0$, and let $\eta \in C_{0}^{\infty}(\mathbb{R})$ be a function supported on $(-1,1) \subset \mathbb{R}$ whose integral over $\mathbb{R}$ is one. Then

$$
e_{\epsilon}(x, t)=\int_{\mathbb{R}} e\left(x, t^{\prime}\right) \eta\left(\frac{t-t^{\prime}}{\epsilon}\right) d t^{\prime}
$$

satisfies

$$
\left.\left(\partial_{t}^{2}+\mathcal{A}\right)\right) e_{\epsilon}=0 \quad \text { in } M \times(\epsilon, T-\epsilon),\left.\quad \partial_{\nu} e_{\epsilon}\right|_{\partial M \times(\epsilon, T-\epsilon)}=0
$$

and $e_{\epsilon} \in C^{\infty}\left((\epsilon, T-\epsilon), H_{0}^{-1}(M)\right)$. By representing $e_{\epsilon}$ in terms of eigenfunctions of $\mathcal{A}$, we see that $e_{\epsilon} \in C^{\infty}\left((\epsilon, T-\epsilon), \mathcal{D}\left(\mathcal{A}^{\infty}\right)\right) \subset C^{\infty}(M \times$ $(\epsilon, T-\epsilon))$. Using Tataru's unique continuation theorem 35 we see that if $0<\epsilon<(T-\operatorname{diam}(M)) / 2$ then $e_{\epsilon}(T / 2)=\partial_{t} e_{\epsilon}(T / 2)=0$. Hence $e_{\epsilon}=0$ identically on $M \times[0, T]$. When $\epsilon \rightarrow 0$, we see that also $e$ vanishes identically and thus $\phi=\psi=0$.

4.1. Proof of Theorem 5. On $X$ we will study the minimization problem

$$
\min _{(h, a) \in X} \mathcal{F}(h, a, \alpha)
$$

where $\alpha \in(0,1)$ and $\mathcal{F}$ is defined in equation (5). By 32, the map $h \mapsto u^{h}$ is continuous $L^{2}(\partial M \times[0, T]) \rightarrow C\left([0, T] ; H^{5 / 6-\epsilon}(M)\right), \epsilon>0$. Thus $(h, a) \mapsto \mathcal{F}(h, a, \alpha)$ is continuous map $X \rightarrow \mathbb{R}$.

Lemma 8. For any $\alpha \in(0,1)$ minimization problem (7) has a unique minimizer $(h, a) \in X$. This minimizer is the unique solution to

$$
(\alpha+L)\left(\begin{array}{l}
h \\
a
\end{array}\right)=\left(\begin{array}{c}
P K f \\
0
\end{array}\right),
$$

where $L$ is defined in equation (3). Furthermore, $L: X \rightarrow X$ is nonnegative, bounded, and selfadjoint. 
Proof. We have

$$
\begin{aligned}
\mathcal{F}(h, a, \alpha)= & \langle f-P h, K(f-P h)\rangle+\langle P h-a, K(P h-a)\rangle \\
& +\left\langle\partial_{t} a, K \partial_{t} a\right\rangle \\
& +\alpha\left(\langle h, h\rangle+\langle a, a\rangle+\left\langle\partial_{t} a, \partial_{t} a\right\rangle\right),
\end{aligned}
$$

Here $K$ and $P$ are selfadjoint in $L^{2}(M)$. The dual $Y^{*}$ of the Hilbert space $Y=H^{1}\left([0,2 T] ; L^{2}(M)\right)$ can be identified with $H^{-1}\left([0,2 T] ; L^{2}(M)\right)$. As $Q$ the inverse of $1-\partial_{t}^{2}$, it can be considered as an operator $Q: Y^{*} \rightarrow Y$. Thus using $\partial_{t} K \partial_{t}: Y \rightarrow Y^{*}$, we can write

$$
\left\langle\partial_{t} a, K \partial_{t} a\right\rangle_{L^{2}(M)}=-\left\langle\partial_{t} K \partial_{t} a, a\right\rangle_{Y^{*}, Y}=-\left\langle Q \partial_{t} K \partial_{t} a, a\right\rangle_{Y}
$$

Thus, using the inner product on $X=L^{2}(M) \times Y$, we can rewrite $\mathcal{F}$ as

$$
\begin{aligned}
\mathcal{F}(h, a, \alpha)= & \langle f, K f\rangle+2\left\langle\left(\begin{array}{l}
h \\
a
\end{array}\right),\left(\begin{array}{ll}
1 & 0 \\
0 & Q
\end{array}\right)\left(\begin{array}{c}
-P K f \\
0
\end{array}\right)\right\rangle_{X} \\
& +\left\langle\left(\begin{array}{l}
h \\
a
\end{array}\right),(\alpha+L)\left(\begin{array}{l}
h \\
a
\end{array}\right)\right\rangle_{X} .
\end{aligned}
$$

As $Q: Y^{*} \rightarrow Y$ and $\partial_{t} K \partial_{t}: Y \rightarrow Y^{*}$ are bounded, $L: X \rightarrow X$ is bounded. A direct calculation shows that $L$ is self-adjoint. Setting $f=0$ and $\alpha=0$ in equation (9) shows that $L$ is non-negative.

Let us next observe that $\mathcal{F}$ is strictly convex, so for the first claim it suffices to prove existence; by convexity, a local minimum is a global minimum, and by strict convexity, there is only one global minimum (see [38, Theorem 38.C]). To prove existence, we recall that by [1], $x \in X$ is a local minimum of $\mathcal{F}: X \rightarrow \mathbb{R}$ provided that:

(i) the first two Fréchet derivatives at $x, D_{x} \mathcal{F}(\xi)$ and $D_{x}^{2} \mathcal{F}(\xi, \eta)$, exist and are continuous,

(ii) $D_{x} \mathcal{F}=0$,

(iii) $u \mapsto D_{x}^{2} \mathcal{F}(\xi, \cdot)$ is a linear isomorphism $X \rightarrow X^{*}$, and

(iv) $D_{x}^{2} \mathcal{F}(\xi, \xi)>0$ for all $\xi \neq 0$.

The sought Fréchet derivatives of $\mathcal{F}: X \rightarrow \mathbb{R}$ are

$$
\begin{aligned}
D \mathcal{F}_{h, a}(\xi) & =2\left\langle\left(\begin{array}{ll}
1 & 0 \\
0 & Q
\end{array}\right)\left(\begin{array}{c}
-P K f \\
0
\end{array}\right)+(\alpha+L)\left(\begin{array}{l}
h \\
a
\end{array}\right), \xi\right\rangle, \\
D^{2} \mathcal{F}_{h, a}(\xi, \eta) & =2\langle\xi,(\alpha+L) \eta\rangle, \quad \xi, \eta \in X .
\end{aligned}
$$

It remains to prove that $\alpha+L$ is invertible, but if $(\alpha+L)(\xi)=0$, then by non-negativity, $0 \leq\langle L \xi, x\rangle_{X}=-\alpha\|\xi\|_{X}^{2} \leq 0$, and $\xi=0$.

Lemma 9. Iteration scheme (41) converges to the unique solution to equation (8).

Proof. Using $S$ and $\omega$ defined in definition 4, we may rewrite equation (8) as

$$
(I-S)\left(\begin{array}{c}
h \\
a
\end{array}\right)=\frac{1}{\omega}\left(\begin{array}{c}
P K f \\
0
\end{array}\right)
$$


For a self-adjoint operator $B$, the norm satisfies $\|B\|=\sup \{|\langle x, B x\rangle|$ : $\|x\|=1\}$. Hence $\alpha+\langle L x, x\rangle<\omega / 2$, and by non-negativity,

$$
\|S\| \leq 1-\frac{\alpha}{\omega}<1
$$

We may therefore iteratively solve $h, a$ by a convergent von Neumann sum.

Lemma 10. Minimizers $h(\alpha), a(\alpha) \in X$ for (7) satisfy

$$
\begin{aligned}
\operatorname{supp} h(\alpha) & \subset B \\
a(\alpha) & \in \operatorname{range} Q, \\
\lim _{\alpha \rightarrow 0}\left(\begin{array}{c}
u^{a(\alpha)}(T) \\
u_{t}^{a(\alpha)}(T)
\end{array}\right) & =\left(\begin{array}{c}
\chi_{N} u^{f}(T) \\
0
\end{array}\right),
\end{aligned}
$$

where all limits are in $L^{2}(M)$.

Proof. The first two claims follow by writing out (8)). For the other results, let us define $Z: X \rightarrow \mathbb{R}$ by

$$
\begin{aligned}
Z(h, a)= & \frac{1}{2}\left\|\chi_{N} u^{f}(T)-u^{P h}(T)\right\|^{2}+\frac{1}{4}\left\|u^{a}(T)-\chi_{N} u^{f}(T)\right\|^{2} \\
& +\left\|u_{t}^{a}(T)\right\|^{2} .
\end{aligned}
$$

To prove the last claim we show that for any $\varepsilon>0$ there exists an $\alpha(\varepsilon) \in(0,1)$ such that $Z(h(\alpha), a(\alpha))<4 \varepsilon$ when $\alpha \in(0, \alpha(\varepsilon))$. Let us note that

$$
u^{P f}(T)=\chi_{N} u^{P f}(T), \quad f \in L^{2}(\partial M,[0, T]) .
$$

Hence, for any $(h, a) \in X$,

$$
\begin{aligned}
\mathcal{F}(P h, a, \alpha)= & \left\|\left(1-\chi_{N}\right) u^{f}(T)\right\|^{2}+\left\|\chi_{N} u^{f}(T)-u^{P h}(T)\right\|^{2} \\
& +\left\|u^{P h}(T)-u^{a}(T)\right\|^{2}+\left\|u_{t}^{a}(T)\right\|^{2} \\
& +\alpha\left(\|h\|^{2}+\|a\|^{2}+\left\|\partial_{t} a\right\|^{2}\right) .
\end{aligned}
$$

It follows that for any $(h, a) \in X$ and $\alpha \in(0,1)$,

$$
Z(P h, a) \leq \mathcal{F}(P h, a, \alpha)-\left\|\left(1-\chi_{N}\right) u^{f}(T)\right\|^{2} .
$$

Here we have estimated the second term in $Z$ using the triangle inequality and the inequality $(s+t)^{2} \leq 2\left(s^{2}+t^{2}\right)$. Let us fix $\varepsilon \in(0,1)$. By Proposition 3 there exists an $h_{\varepsilon} \in L^{2}(B)$ such that

$$
\left\|\chi_{N} u^{f}(T)-u^{P h_{\varepsilon}}(T)\right\|^{2}<\varepsilon,
$$

and by Proposition 2 there exists an $a_{\varepsilon} \in H^{1}(B)$ such that

$$
\begin{aligned}
\left\|u^{a_{\varepsilon}}(T)-\chi_{N} u^{P h_{\varepsilon}}(T)\right\|^{2} & <\varepsilon, \\
\left\|u_{t}^{a_{\varepsilon}}(T)\right\|^{2} & <\varepsilon .
\end{aligned}
$$

As $h_{\varepsilon}=P h_{\varepsilon}$ we have

$\mathcal{F}\left(h_{\varepsilon}, a_{\varepsilon}, \alpha\right)=\left\|\left(1-\chi_{N}\right) u^{f}(T)\right\|^{2}+3 \varepsilon+\alpha\left(\left\|h_{\varepsilon}\right\|^{2}+\left\|a_{\varepsilon}\right\|^{2}+\left\|\partial_{t} a_{\varepsilon}\right\|^{2}\right)$, 
and if $\alpha \in(0, \alpha(\varepsilon))$, where

$$
\alpha(\epsilon)=\frac{\varepsilon}{1+\left\|h_{\varepsilon}\right\|^{2}+\left\|a_{\varepsilon}\right\|^{2}+\left\|\partial_{t} a_{\varepsilon}\right\|^{2}},
$$

then the minimizer $h(\alpha), a(\alpha)$ of $\mathcal{F}$ satisfies

$$
\begin{aligned}
Z(h(\alpha), a(\alpha)) & \leq \mathcal{F}(P h(\alpha), a(\alpha), \alpha)-\left\|\left(1-\chi_{N}\right) u^{f}(T)\right\|^{2} \\
& \leq \mathcal{F}\left(h_{\varepsilon}, a_{\varepsilon}, \alpha\right)-\left\|\left(1-\chi_{N}\right) u^{f}(T)\right\|^{2} \\
& <4 \varepsilon .
\end{aligned}
$$

4.2. Proof of Theorem 7. Let us note that for any $\varepsilon>0$, then $\Gamma_{k} \subset B(\widehat{z}, \varepsilon)$ for sufficiently large $k$. By Theorem 15, the following limits exist in $Y$,

$$
\begin{aligned}
a(\alpha, \varepsilon) & =\lim _{n \rightarrow \infty} a_{n}(\alpha, \varepsilon), \\
a(\alpha, j, \varepsilon) & =\lim _{n \rightarrow \infty} a_{n}(\alpha, j, \varepsilon) .
\end{aligned}
$$

and

$$
\begin{aligned}
\lim _{\alpha \rightarrow 0}\left(\begin{array}{c}
u^{a(\alpha, \varepsilon)}(T) \\
u_{t}^{a(\alpha, \varepsilon)}(T)
\end{array}\right) & =\left(\begin{array}{c}
\chi_{N(\varepsilon)} u^{f}(T) \\
0
\end{array}\right), \\
\lim _{\alpha \rightarrow 0}\left(\begin{array}{l}
u^{a(\alpha, j, \varepsilon)}(T) \\
u_{t}^{a(\alpha, j, \varepsilon)}(T)
\end{array}\right) & =\left(\begin{array}{c}
\chi_{N(j, \varepsilon)} u^{f}(T) \\
0
\end{array}\right),
\end{aligned}
$$

where

$$
\begin{aligned}
N(\varepsilon) & =M(\partial M, \widehat{T}-\varepsilon), \\
N(j, \varepsilon) & =M(\partial M, \widehat{T}-\varepsilon) \cup M\left(\Gamma_{j}, \widehat{T}+\varepsilon\right) .
\end{aligned}
$$

We define $b(\alpha, j, \varepsilon)=\lim _{n \rightarrow \infty} b_{n}(\alpha, j, \varepsilon)$, whence

$$
b(\alpha, j, \varepsilon)=\varepsilon^{-\frac{m+1}{2}}(a(\alpha, j, \varepsilon)-a(\alpha, \varepsilon)) .
$$

Lemma 11. In $L^{2}(M)$,

$$
\lim _{j \rightarrow \infty} \lim _{\alpha \rightarrow 0}\left(\begin{array}{c}
u^{b(\alpha, j, \varepsilon)}(T) \\
u_{t}^{b(\alpha, j, \varepsilon)}(T)
\end{array}\right)=\varepsilon^{-\frac{m+1}{2}}\left(\begin{array}{c}
\chi_{J(\varepsilon)} u^{f}(T) \\
0
\end{array}\right),
$$

where

$$
J(\varepsilon)=M(\widehat{z}, \widehat{T}+\varepsilon) \backslash M(\partial M, \widehat{T}-\varepsilon) .
$$

Proof. Since $a \mapsto u^{a}$ is linear, it suffices to prove that pointwise

$$
\lim _{j \rightarrow \infty} \chi_{M\left(\Gamma_{j}, \widehat{T}+\varepsilon\right) \backslash M(\partial M, \widehat{T}-\varepsilon)}(x)=\chi_{J(\varepsilon)}(x), \quad x \in M .
$$

This is clear for $x \in J(\varepsilon)$. If $x \notin J(\varepsilon)$ we claim that $x \notin M\left(\Gamma_{j}, \widehat{T}+\varepsilon\right)$ for large $j$. However, if $d(x, \widehat{z})>\widehat{T}+\varepsilon$, then

$$
\Gamma_{l} \subset B\left(\widehat{z}, \frac{d(x, \widehat{z})-\widehat{T}-\varepsilon}{2}\right)
$$


for large $l$. For $y \in \Gamma_{l}, d(x, y) \geq d(x, \widehat{z})-d(y, \widehat{z})>\widehat{T}+\varepsilon$, so $d\left(x, \Gamma_{l}\right)>\widehat{T}+\varepsilon$, and $x \notin M\left(\Gamma_{l}, \widehat{T}+\varepsilon\right)$.

The next Lemma show that $J(\varepsilon)$ are sets that shrink onto $\widehat{x}$ in the case when $\widehat{T}<\tau(\widehat{z})$.

Lemma 12 (Properties of $J(\delta))$. For any $\varepsilon>0$, there is a $\delta>0$ such that

$$
J(\delta) \subset B(\widehat{x}, \varepsilon) .
$$

Moreover, if $\widehat{T}<\tau(\widehat{z})$, then $\{\widehat{x}\} \subset J(\delta)$ for all $\delta$ implying that $\bigcap_{\delta>0} J(\delta)=\{\widehat{x}\}$. If $\widehat{T}>\tau(\widehat{z})$ then $J(\delta)=\emptyset$ for $\delta$ small enough.

Proof. Let us first prove (12). For a contradiction suppose that $\varepsilon>0$ and $x_{1}, x_{2}, \ldots$ is a sequence such that

$$
x_{j} \in J(1 / j), \quad x_{j} \notin B(\widehat{x}, \varepsilon) .
$$

As $M$ is compact, we can move onto a subsequence and assume that $x_{j}$ converges to an $x \in M \backslash B(\widehat{x}, \varepsilon)$. Now $d\left(x_{j}, \widehat{z}\right) \leq \widehat{T}+1 / j$ and $d\left(x_{j}, \partial M\right)>\widehat{T}-1 / j$, and as $x \mapsto d(x, \partial M)$ is continuous,

$$
d(x, \widehat{z}) \leq \widehat{T}, \quad d(x, \partial M) \geq \widehat{T} .
$$

Thus $\widehat{T} \leq d(x, \partial M) \leq d(x, \widehat{z}) \leq \widehat{T}$. If $\widehat{T}>\tau(\widehat{z})$, we have $d(\widehat{x}, \partial M)<$ $\widehat{T}$ and obtain a contradiction. Thus we can assume that $\widehat{T} \leq \tau(\widehat{z})$. Then the above inequalities yield that $d(x, \widehat{z})=d(x, \partial M)=\widehat{T}$. As $M$ is compact, there is a geodesic from $\widehat{z}$ to $x$ that realizes $d(\widehat{x}, \widehat{z})$. Then $\eta$ also realizes $d(\widehat{x}, \partial M)$, and $\eta$ must necessarily be normal to $\partial M$ 14. Thus $\eta=\gamma$, and $\widehat{x}=x$; a contradiction. Thus (12) is proven.

If $\widehat{T}<\tau(\widehat{z})$, then clearly $\widehat{x} \in J(\delta)$ for all $\delta>0$. On the other hand, if $\widehat{T}>\tau(\widehat{z})$ then there is $z^{\prime} \in \partial M$ such that $d\left(\widehat{x}, z^{\prime}\right)<d(\widehat{x}, \widehat{z})$ and we see that $\widehat{x} \notin J(\delta)$ for small $\delta$. Thus we have shown that $J(\delta)=\emptyset$ for $\delta$ small enough.

Proof of Theorem 1 . Consider first the case when $\widehat{T}<\tau(\widehat{z})$. Then we observe that the following limit exists

$$
C(\widehat{x})=\lim _{\varepsilon \rightarrow 0} \frac{\operatorname{Vol}(J(\varepsilon))}{\varepsilon^{\frac{m+1}{2}}}
$$

exists (see [11]). Here $\operatorname{Vol}(A)=\int_{A} 1 \mathrm{~d} V$ when $A \subset M$. Let us also note that $B(\widehat{x}, \varepsilon / 2) \subset J(\varepsilon)$ so $\operatorname{Vol}(J(\varepsilon))>0$. Thus, as $u^{f}(T, \cdot)$ is continuous,

$$
\begin{aligned}
\lim _{\varepsilon \rightarrow 0}\left\langle\frac{1}{\varepsilon^{\frac{m+1}{2}}} \chi_{J(\varepsilon)} u^{f}(T), \phi\right\rangle & =C(\widehat{x}) \lim _{\varepsilon \rightarrow 0} \frac{1}{\operatorname{Vol}(J(\varepsilon))} \int_{J(\varepsilon)} u^{f}(T, x) \phi(x) \mathrm{d} V(x) \\
& =\left\langle C(\widehat{x}) u^{f}(T, \widehat{x}) \delta_{\widehat{x}}, \phi\right\rangle, \quad \phi \in \mathscr{D}(M) .
\end{aligned}
$$

The result follows by [21, Theorem 2.1.8]. 
In the case when $\widehat{T}>\tau(\widehat{z}), J(\epsilon)=\emptyset$ for $\epsilon$ small enough, and thus the limits (10) and (11) are the same. Hence limit (6) is zero.

\section{ACKNOWLEDGEMENTS}

The research has been partially supported by Tekes project MASIT03, EPSRC, and the Academy of Finland Center of Excellence programme 213476.

\section{REFERENCES}

[1] R. Abraham, J.E. Marsden, T.Ratiu, Manifolds, Tensor Analysis, and Applications, (Applied Mathematical Sciences), Springer, 2001.

[2] M. Anderson, A. Katsuda, Y. Kurylev, M. Lassas, M. Taylor: Boundary regularity for the Ricci equation, Geometric Convergence, and Gel'fand's Inverse Boundary Problem, Invent. Math. 158 (2004), 261-321.

[3] C. Bardos: A mathematical and deterministic analysis of the time-reversal mirror. Inside out: inverse problems and applications, 381-400, Math. Sci. Res. Inst. Publ., 47, Cambridge Univ. Press, 2003.

[4] C. Bardos, M. Fink: Mathematical foundations of the time reversal mirror. Asymptot. Anal. 29 (2002), 157-182.

[5] G. Bal, O. Pinaud: Time reversal based detection in random media, Inverse Problems, 21(2005), 1593-1620.

[6] B. Bal, L.Ryzhik: Time Reversal for Classical Waves in Random Media, C. R. Acad. Sci. Paris, Serie I, 333 (2001), 1041-1046

[7] G. Bal, L.Ryzhik: Time Reversal and refocusing in Random Media, SIAM J. Appl. Math 63 (2003), 1475-1498.

[8] M. Belishev: An approach to multidimensional inverse problems for the wave equation. (Russian) Dokl. Akad. Nauk SSSR 297 (1987), 524-527.

[9] M. Belishev: Boundary control in reconstruction of manifolds and metrics (the BC method). Inverse Problems 13 (1997), R1-R45.

[10] M. Belishev, Y. Kurylev: To the reconstruction of a Riemannian manifold via its spectral data (BC-method). Comm. Partial Differential Equations 17 (1992), $767-804$.

[11] K. Bingham, Y. Kurylev, M. Lassas, S. Siltanen: Iterative time-reversal control for inverse problems, to appear in Inverse Problems and Imaging, preprint: arXiv:math/0701133.

[12] L. Borcea, G. Papanicolaou, C. Tsogka, J. Berryman: Imaging and time reversal in random media. Inverse Problems 18 (2002), 1247-1279.

[13] L. Borcea, G. Papanicolaou, C. Tsogka: Theory and applications of time reversal and interferometric imaging. Inverse Problems, 19 (2003), 5139-5164

[14] I. Chavel: Riemannian geometry. A modern introduction. Cambridge University Press, 2006. xvi $+471 \mathrm{pp}$.

[15] M. Cheney, D. Isaacson, M. Lassas: Optimal acoustic measurements. SIAM J. Appl. Math. 61 (2001), 1628-1647.

[16] D. Cassereau, M. Fink Time-reversal focusing through a plane interface separating two fluids J. Acoust. Soc. Am., 96 (1994), 3145-3154.

[17] M. Fink: Time reversal mirrors. J. Phys. D: Appl.Phys. 26 (1993), 1333-1350.

[18] M. Fink: Time reversal of Ultrasonic fields. IEEE Transactions on ultrasonics, Ferroelectrics, and Frequency control, Vol. 39, No. 5, Sep. 1992, Part I (pp. 555566), Part II (pp. 567-578), Part III (pp. 579-592) 
[19] M. Fink, D. Cassereau, A. Derode, C. Prada, P. Roux, M. Tanter, J.-L. Thomas, F. Wu: Time-reversed acoustics. Rep. Prog. Phys. 63 (2000), 1933-1995. [20] M. Fink Time-reversal acoustics in complex environments Geophysics, 71 (2006), SI151-SI164.

[21] L. Hörmander, The analysis of linear partial differential operators. I., Springer, 1990.

[22] L. Hörmander, The analysis of linear partial differential operators. IV. Grundlehren der Mathematischen Wissenschaften, 275. Springer, 1985. vii +352 pp.

[23] D. Isaacson: Distinguishability of conductivities by electric current computed tomography. IEEE Trans. on Medical Imaging MI-5 (1986), 92-95.

[24] B.L.G. Jonsson, M. Gustafsson, V.H. Weston, M.V. de Hoop: Retrofocusing of acoustic wave fields by iterated time reversal. SIAM J. Appl. Math. 64 (2004), $1954-1986$.

[25] A. Katsuda, Y. Kurylev, M. Lassas: Stability of boundary distance representation and reconstruction of Riemannian manifolds, Inverse Problems and Imaging 1 (2007), 135-157.

[26] A. Katchalov, Y. Kurylev: Multidimensional inverse problem with incomplete boundary spectral data. Comm. Part. Diff. Equations 23 (1998), 55-95.

[27] A. Katchalov, Y. Kurylev, M. Lassas: Inverse boundary spectral problems. Chapman \& Hall/CRC, 2001. xx + 290 pp.

[28] A. Katchalov, Y. Kurylev, M. Lassas: Energy measurements and equivalence of boundary data for inverse problems on non-compact manifolds. Geometric methods in inverse problems and PDE control (eds. C. Croke, I. Lasiecka, G. Uhlmann, M. Vogelius), 183-213, IMA volumes in Mathematics and Applications, 137, Springer, 2004.

[29] A. Katchalov, Y. Kurylev, M. Lassas, N. Mandache: Equivalence of timedomain inverse problems and boundary spectral problem, Inverse problems 20 (2004), 419-436.

[30] M. Klibanov, A. Timonov: On the mathematical treatment of time reversal. Inverse Problems 19 (2003), 1299-1318.

[31] Y. Kurylev, M. Lassas: Hyperbolic inverse boundary-value problem and timecontinuation of the non-stationary Dirichlet-to-Neumann map. Proc. Roy. Soc. Edinburgh Sect. A 132 (2002), 931-949.

[32] I. Lasiecka, R. Triggiani: Sharp regularity theory for second order hyperbolic equations of Neumann type. I. $L_{2}$ nonhomogeneous data. Ann. Mat. Pura Appl. 157 (1990), 285-367.

[33] C. Prada, M. Fink: Eigenmodes of the time reversal operator: A solution to selective focusing in multiple-target media. Wave Motion 20 (1994), 151-163.

[34] C. Prada, J.-L. Thomas, M. Fink: The iterative time reversal process: Analysis of the convergence. J. Acoust. Soc. Am. 97 (1995), 62-71.

[35] D. Tataru: Unique continuation for solutions to PDEs, between Hörmander's theorem and Holmgren's theorem. Comm. Part. Diff. Equations 20 (1995), 855884.

[36] D. Tataru: On the regularity of boundary traces for the wave equation. Ann. Scuola Norm. Sup. Pisa Cl. Sci. 26 (1998), 185-206.

[37] D. Tataru: Unique continuation for operators with partially analytic coefficients. J. Math. Pures Appl. 78 (1999), 505-521.

[38] E. Zeidler, Nonlinear functional analysis and its applications III, Variational Methods and Optimization, Springer, 1984

[39] M. Fink, J. De Rosny Time reversal experiments in random media and in chaotic cavities Nonlinearity, 15 (2002), 1-18. 
Matias Dahl and Matti lassas, Institute of Mathematics, P.O.Box 1100, 02015 Helsinki University of Technology, Finland

Anna Kirpichnikova, School of Mathematics, The University of Edinburgh, JCMB Mayfield Road Edinburgh EH9 3JZ, UK 